\title{
A NEW METHOD OF FINDING THE EQUATION OF A RATIONAL PLANE CURVE FROM ITS PARAMETRIC EQUATIONS.
}

\author{
BY JOSEPH EUGENE ROWE.
}

(Read before the American Mathematical Society, December 27, 1915.)

IN this paper we shall give a new method of deriving the equation of a rational plane curve from its parametric equations.

Let the parametric equations of the rational plane curve $R^{n}$ of order $n$ be

$$
x_{i}=a_{i} t^{n}+{ }^{n} C_{1} b_{i} t^{n-1}+{ }^{n} C_{2} c_{i} t^{n-2}+\cdots \quad(i=0,1,2) .
$$

In particular if $n=2$, equations (1) become

$$
x_{i}=a_{i} t^{2}+2 b_{i} t+c_{i} \quad(i=0,1,2),
$$

which are the parametric equations of a conic or $R^{2}$. The coordinates of the points of the $R^{2}$ whose parameters are $t_{1}$ and $t_{2}$ are $a_{i} t_{1}^{2}+2 b_{i} t_{1}+c_{i}$ and $a_{i} t_{2}^{2}+2 b_{i} t_{2}+c_{i}$, where $i=0,1,2$. Hence the equation of the line on $t_{1}$ and $t_{2}$, written out in full, is

(3) $\left|\begin{array}{ccc}a_{0} t_{1}^{2}+2 b_{0} t_{1}+c_{0} & a_{1} t_{1}^{2}+2 b_{1} t_{1}+c_{1} & a_{2} t_{1}^{2}+2 b_{2} t_{1}+c_{2} \\ a_{0} t_{2}^{2}+2 b_{0} t_{2}+c_{0} & a_{1} t_{2}^{2}+2 b_{1} t_{2}+c_{1} & a_{2} t_{2}^{2}+2 b_{2} t_{2}+c_{2} \\ x_{0} & x_{1} & x_{2}\end{array}\right|=0$.

By subtracting the second row from the first and removing the factor $t_{1}-t_{2}$, equation (3) reduces to

$$
\left|\begin{array}{ccc}
a_{0}\left(t_{1}+t_{2}\right)+2 b_{0} & a_{1}\left(t_{1}+t_{2}\right)+2 b_{1} & a_{2}\left(t_{1}+t_{2}\right)+2 b_{2} \\
a_{0} t_{2}{ }^{2}+2 b_{0} t_{2}+c_{0} & a_{1} t_{2}{ }^{2}+2 b_{1} t_{2}+c_{1} & a_{2} t_{2}{ }^{2}+2 b_{2} t_{2}+c_{2} \\
x_{0} & x_{1} & x_{2}
\end{array}\right|=0 .
$$

The expanded form of (4) is

$$
2|a b x| * t_{1} t_{2}+|a c x|\left(t_{1}+t_{2}\right)+2|b c x|=0 \text {. }
$$

* $\mathrm{By}|a b x|$ is meant the three-rowed determinant $\left|\begin{array}{lll}a_{0} & b_{0} & x_{c} \\ a_{1} & b_{1} & x_{1} \\ a_{2} & b_{2} & x_{2}\end{array}\right|$. 
If two values are assigned to $t_{1}$ and $t_{2}$, (5) is the equation of the line which cuts out of the $R^{2}$ the points whose parameters are $t_{1}$ and $t_{2}$. If the coordinates of a particular point are assigned to the $x_{i}$ and a particular value of $t_{1}$ is given, (5) yields a value of $t_{2}$ which is the parameter of the other point cut out of the $R^{2}$ by the line on $x_{i}$ and $t_{1}$. So long as $x_{i}$ and $t_{1}$ are distinct points a definite value of $t_{2}$ may be obtained from (5). But if $x_{i}$ moves up and coincides with the point on the $R^{2}$ whose parameter is $t_{1}$, the value of $t_{2}$ is arbitrary. With these facts in mind it is desirable to arrange (5) in the form

$$
\left[2|a b x| t_{1}+|a c x|\right] t_{2}+\left[|a c x| t_{1}+2|b c x|\right]=0 .
$$

The value of $t_{2}$ in (6) is arbitrary if only the two equations

$$
2|a b x| t_{1}+|a c x|=0, \quad|a c x| t_{1}+2|b c x|=0
$$

are consistent, and the necessary and sufficient condition that these two equations (7) be consistent is

$$
\left|\begin{array}{rr}
2|a b x| & |a c x| \\
|a c x| & 2|b c x|
\end{array}\right|=0 .
$$

That is, (8) is the equation of the $R^{2}$, for it is the locus of a point $x_{i}$ subject to the condition that a line on this point and a point of the $R^{2}$ fails to determine a second point of the $R^{2}$, or in other words, it is the locus of a point $x_{i}$ which coincides with a point of the $R^{2}$.

If $n=3$ in (1), we obtain the parametric equations of an $R^{3}$ and the equation of the line determined by two points of the $R^{3}$ may be found in exactly the same way. With the above facts in mind the equation of this line may be thrown into the form

$$
\begin{aligned}
& {\left[3|a b x| t_{1}{ }^{2}+3|a c x| t_{1}+|a d x|\right] t_{2}{ }^{2}} \\
& \quad+\left[3|a c x| t_{1}{ }^{2}+(|a d x|+9|b c x|) t_{1}+3|b d x|\right] t_{2} \\
& +\left[|a d x| t_{1}{ }^{2}+3|b d x| t_{1}+3|c d x|\right]=0 .
\end{aligned}
$$

This line cuts out of the $R^{3}$ the points whose parameters are $t_{1}$ and $t_{2}$. If $t_{1}$ is given and the coordinates of a particular point are assigned to the $x_{i}$, (9) is a quadratic in $t_{2}$ whose roots are the parameters of the other two points cut out of the $R^{3}$ by the line on $t_{1}$ and $x_{i}$. These two values may be found from (9) so long as $x_{i}$ and $t_{1}$ are distinct points, but the values of $t_{2}$ become arbitrary as soon as $t_{1}$ and $x_{i}$ coincide. The neces- 
sary and sufficient condition that the quadratic (9) be satisfied by any two values of $t_{2}$ is expressed by equating to zero the coefficient of each power of $t_{2}$ in (9). The determinant of these three equations equated to zero is

$$
\left|\begin{array}{ccc}
3|a b x| & 3|a c x| & |a d x| \\
3|a c x| & |a d x|+9|b c x| & 3|b d x| \\
|a d x| & 3|b d x| & 3|c d x|
\end{array}\right|=0,
$$

and this is the equation of the $R^{3}$ as the locus of a point $x_{i}$ which coincides with a point of the $R^{3}$.

The method of finding the equation of the line determined by the points of the $R^{n}$ whose parameters are $t_{1}$ and $t_{2}$ requires no formal statement. If this equation is represented symbolically by

$$
n|a b x| t_{1}{ }^{n-1} t_{2}{ }^{n-1} \cdots=0,
$$

by arranging (11) as a binary $(n-1)$-ic in $t_{2}$ and equating the coefficients of the powers of $t_{2}$ to zero $n$ equations are obtained, and the determinant of these equations equated to zero is the equation of the $R^{n}$.

Pennsylvania State College, November, 1915.

\section{THE PHYSICIST J. B. PORTA AS A GEOMETER.}

BY PROFESSOR GINO LORIA.*

IN his remarkable and entertaining Budget of Paradoxes, of which a new edition has just been published under the editorship of David Eugene Smith, Professor De Morgan speaks of the work entitled "Io. Baptista Portae Neapolitani Elementorum curvilineorum Libri tres. In quibus altera de Geometriae parte restituta, agitur de Circuli Quadratura (Romæ, MDCX)," in the following words: "This is a ridiculous attempt, which defies description, except that it is all about lunules."

Such a scornful judgment forms an evident contrast to the opinion expressed by M. Chasles, who in his Apercu Historique ${ }^{+}$

* Translated from the author's manuscript by Madelaine A. Batta.

+ Second edition, Paris, 1875, p. 216. 